\title{
THE INFLUENCE OF THERMAL EXPANSION OF UNBONDED FOUNDRY SANDS ON THE DEFORMATION OF RESIN BONDED CORES
}

\begin{abstract}
Depending on the preparation and the applied materials, moulds and cores can be of high rigidity or can be flexible. Although, chemically bonded moulding materials have relatively good flexibility, their high temperature behaviour determines the dimensional accuracy, the stresses in the castings and can induce several casting defects, such as rattail, veining, etc. The phenomenon is based on two major effects: the thermal expansion of the unbonded foundry sands and the deformation of the sand mixtures. The main objective of the present work was to study the relationship between these two effects, and to improve the knowledge related to the thermo-mechanical interactions between the casting and the mould. Dilatometric analysis of unbonded sand samples were performed and compared to the results of hot distortion tests of moulding mixture specimens. The results showed, that the thermal expansion of foundry sand largely influences the hot distortion behaviour, but depending on the type of binder used.
\end{abstract}

Keywords: foundry sand, hot distortion, moulding material, phenolic resin, thermal expansion

\section{Introduction}

Several types of refractory materials are used in foundry technology based on the desired properties of the mould and core e.g. low expansion, high thermal stability. The most common is silica sand due to its abundance and durability, although it has the highest thermal expansion compared to other types. For steel casting chromite and olivine sands are extensively used since they have high melting point and higher cooling capacity compared to silica. Zircon sand has high dimensional stability. Therefore, it is an adequate base material for investment casting shells. It can also be added to the core sand to decrease expansion and to improve dimensional accuracy.

Foundrymen take the thermal expansion of foundry sands into account in all aspects of the casting manufacturing process; this includes the initial geometry design of the product, the geometrical interpretation of the mould and core making tools, and the solidification of the liquid metal. On the other hand, the deformation of the mixture is a more complex phenomenon and indeed has more effect on the defect formation processes. These effects are overlapping during the casting, however they are traditionally mentioned and studied separately in the literature. Therefore, this work aims to interconnect the knowledge about the thermal expansion of the unbonded sand with the thermo-mechanical deformation of the moulds and cores, since the flexibility of the moulding materials is also influenced by the decomposition of binders.

\section{Materials and methods}

\subsection{Investigated materials}

Four common types of basic foundry sands with different properties were investigated: silica, chromite, olivine and zircon sand. They have various areas of applications outside foundry purposes [1]. These minerals also have a wide range of thermal expansion [2], which gives better opportunities to study the influence of the refractory on the distortion of the specimens. Grain shapes of these refractories were defined by comparing optical microscope images shown in (Fig. 1a-d) with a chart found in literature [3]. Specific surface area of the different sand types was determined by a testing apparatus which operates on air permeability method. Medium grain size (d50) corresponds to the value of the particle diameter at $50 \%$ in the cumulative distribution $[4,5]$.

Results of granulometric analyses (Table 1) (Fig. 2a-d) show that the properties of the various types of refractories are quite different. Zircon sand has the highest surface area due to its rounded grain shape, high sphericity and small medium grain size. Cores made of this refractory have very low permeability, thus usually it is mixed with silica sand. Chromite sand has the lowest surface area since it has the highest medium grain size. The difference between olivine and silica sand is not significant, but the latter is finer and the shape is more rounded.

\footnotetext{
UNIVERSITY OF MISKOLC, DEPARTMENT OF FOUNDRY TECHNOLOGY, MISKOLC, HUNGARY

** JÖNKÖPING UNIVERSITY SCHOOL OF ENGINEERING, DEPARTMENT OF MATERIALS AND MANUFACTURING, JÖNKÖPING, SWEDEN 

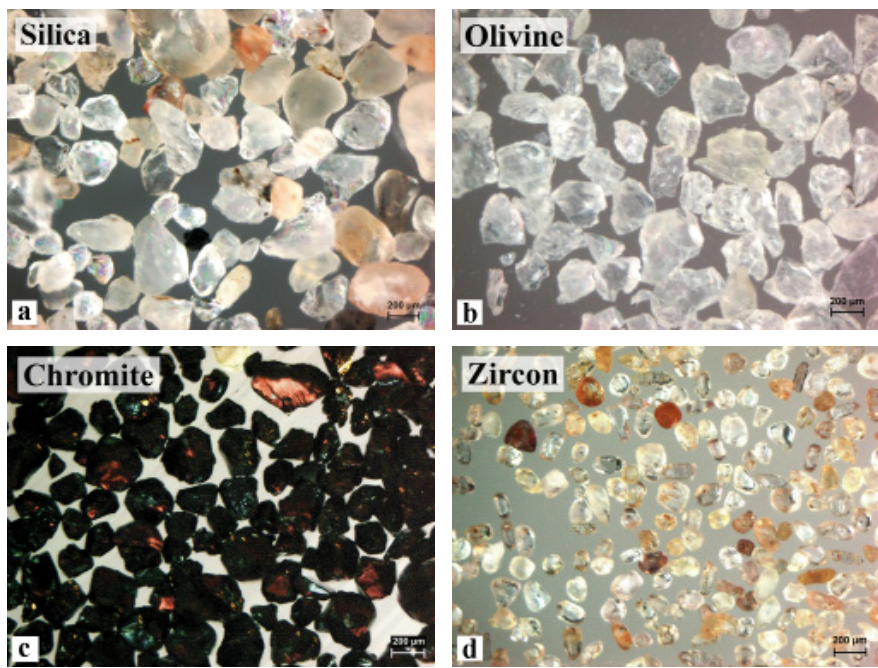

Fig. 1. Optical microscope images of the studied silica (a), olivine (b), chromite (c) and zircon (d) sands
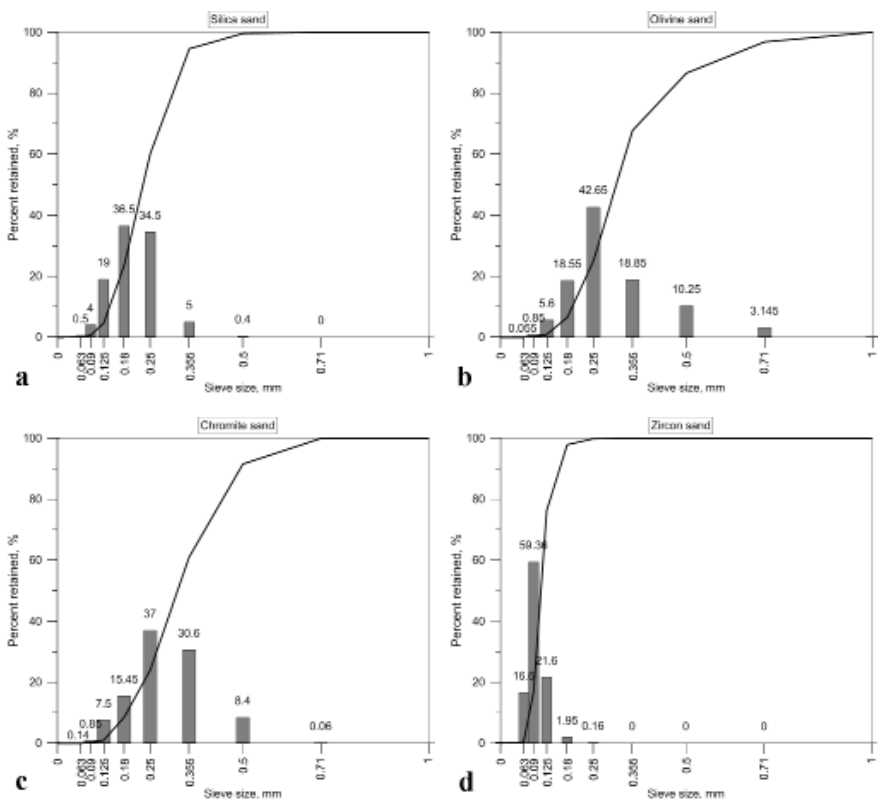

Fig. 2. Grain size distribution of the studied silica (a), olivine (b), chromite (c) and zircon (d) sands

TABLE 1

Granulometric properties of the sand samples

\begin{tabular}{|c|c|c|c|}
\hline \hline Sand type & Grain shape & $\begin{array}{c}\text { Medium grain } \\
\text { size d50, } \mathbf{~ m m}\end{array}$ & $\begin{array}{c}\text { Specific surface } \\
\text { area, } \mathbf{~ c m}^{\mathbf{2}} \mathbf{g}\end{array}$ \\
\hline Silica & Sub-Rounded & 0,23 & 147 \\
\hline Olivine & Angular & 0,31 & 138 \\
\hline Chromite & Sub-Angular & 0,32 & 94 \\
\hline Zircon & Rounded & 0,1 & 237 \\
\hline
\end{tabular}

\subsection{Experimental methods}

Thermal expansion of refractories without binders and additives was measured by a Netzsch DIL 402 C type horizontal dilatometer. The preparation method was identical in case of every sample. Constant mass of unbonded sand was filled in alumina $\left(\mathrm{Al}_{2} \mathrm{O}_{3}\right)$ crucibles and compacted slightly by vibration. The heating rate was set to $10^{\circ} \mathrm{C} / \mathrm{min}$, the maximum temperature was $1100^{\circ} \mathrm{C}$. In order obtain additional information compared to the literature $[2,6-8]$, the volume change was registered not only during heating up, but also during cooling down in the function of temperature. The measurements were first performed by $\mathrm{Al}_{2} \mathrm{O}_{3}$ standard with the same layout to obtain a correction file containing the expansion of the system and the sample holder.

Regarding to the planning of core deformation tests, results from earlier authors [9-12] were reviewed and considered. The hot distortion test apparatus was first developed by the British Cast Iron Association (BCIRA) in the seventies. Z. Ignaszak modified the original apparatus with supplementary devices, such as additional gas heating, pyrometer and thermovison camera. The state-of-the-art method known as the Hot Distortion Plus ( ensures temperature monitoring and thermo-mapping of the specimen during the displacement tests, allowing the opportunity to obtain valuable additional information for the simulation of the phenomena. Based on the limited availability, hot distortion tests in this paper were performed by a Simpson-Gerosa hot distortion tester [13]. The samples were made of the studied refractories mixed with furan urea and phenolic resins chosen by their different thermal behaviour. Table 2 contains detailed information about the mixture compositions.

TABLE 2

Production parameters of resin bonded samples

\begin{tabular}{|c|c|c|c|}
\hline \hline $\begin{array}{c}\text { Binder } \\
\text { type }\end{array}$ & $\begin{array}{c}\text { Binder amount } \\
\text { (in respect of } \\
\text { sand mass), \% }\end{array}$ & $\begin{array}{c}\text { Hardener amount } \\
\text { (in respect of } \\
\text { resin mass), \% }\end{array}$ & $\begin{array}{c}\text { Type of } \\
\text { process }\end{array}$ \\
\hline Furan urea & 1,6 & 0,32 & Hot-box \\
\hline Phenolic & 1,6 & 0,32 & Hot-box \\
\hline
\end{tabular}

Standard specimens with dimensions $114,3 \times 25,4 \times 6,35 \mathrm{~mm}$ were produced by a laboratory core shooting machine. The samples were fixed on one end and the middle was heated by a gas burner to ensure high heat exchange coefficient between the flame and the sample. Flame temperature was approximately $900^{\circ} \mathrm{C}$. Because of the layout of the applied instrument and the current primary focus on the volume changes of both unbonded sands and mixtures, displacement was registered only in the function of time, temperature of the samples was not recorded. Three

TABLE 3

Density of the refractories and bulk density values of the studied specimens

\begin{tabular}{|c|c|c|c|}
\hline \hline Sand type & $\begin{array}{c}\text { Density, } \\
\mathbf{g} / \mathbf{c m}^{\mathbf{3}}\end{array}$ & $\begin{array}{c}\text { Bulk density of un- } \\
\text { bonded sample, } \mathbf{g} / \mathbf{c m}^{\mathbf{3}}\end{array}$ & $\begin{array}{c}\text { Bulk density of resin } \\
\text { bonded sample, } \mathbf{g} / \mathbf{c m}^{\mathbf{3}}\end{array}$ \\
\hline Silica & 2,65 & 1,5 & 1,51 \\
\hline Olivine & 3,3 & 1,8 & 1,82 \\
\hline Chromite & 4,4 & 2,49 & 2,51 \\
\hline Zircon & 4,7 & 2,6 & 2,63 \\
\hline
\end{tabular}


samples were measured from each type of materials during the hot distortion tests and during the dilatometric studies as well.

The bulk density values of unbonded and resin bonded samples were set to fit the actual difference in the density values of the various types of minerals studied. Additionally, the bulk density of the samples used for dilatometric and hot distortion measurements were approximately equal with respect to the same type of refractory (Table 3 ).

\section{Results and discussion}

Fig. 3a-d show significant differences between the maximum thermal expansions of various types of sands. Due to the allotropic transformation of quartz at $573^{\circ} \mathrm{C}$ silica sand reaches a maximum thermal expansion of 2,2-2,5\% (Fig. 3a). After a shrinking period a secondary peak occurs at approximately $950^{\circ} \mathrm{C}$. According to earlier authors [6] the reason for this secondary peak is unknown, but can have a contribution in eliminating veining defects. During the cooling of the samples, the volume is relatively constant until $573^{\circ} \mathrm{C}$. Below this temperature, a noticeable shrinkage appears referring to the reversible process taking place in the quartz. However, this shrinkage eventuates a lower degree of volume change, compared to the expansion process during heating up. This effect is due to the irreversible phase transitions of silica at high temperatures. As a result, silica sand has a residual thermal expansion of 0,7 to $1,2 \%$. The maximum expansion of olivine sand up to $1100^{\circ} \mathrm{C}$ is much lower as compared to the silica, approximately $1,2 \%$ (Fig. $3 b$ ). Olivine also has residual thermal expansion of 0,3 to $0,4 \%$. According to Fig. 3c,d chromite and zircon sand have the lowest maximums, around $1 \%$. Contrarily to other types, chromite sand appeared not to have residual thermal expansion, but the results indicate negative expansion.

The reason for that, is that the particles may change their position on each other during the expansion. During cooling, the volume of the sample decreases, the sand grains are shrinking, but these particles are not ensured to find their original position. Since a pushrod applies force on the end of the sample in the horizontal dilatometer, it can dispose the particles to get into a more compacted layout. The measurement starts from the original compacted length of the sample (it is taken as zero point), so any kind of shrinking or compacting compared to it is measured as negative expansion. Zircon sand showed residual thermal expansion rates similar to the olivine. Besides the maximum values, volume change characteristics of the various types of sands are also different, which is expected to have an impact on the hot deformation of mixtures as well.

Understanding of the hot distortion curves is difficult, because they are originated from several different types of processes occurring simultaneously. According to earlier authors [14-16], the deformation is also depending on the properties of the moulding mixture (e.g. properties of the refractory, binder type and amount). Expansion of the basic refractory takes place layer by layer due to the one-sided heating and induces the bending of the specimen. The binder decomposes simultaneously,
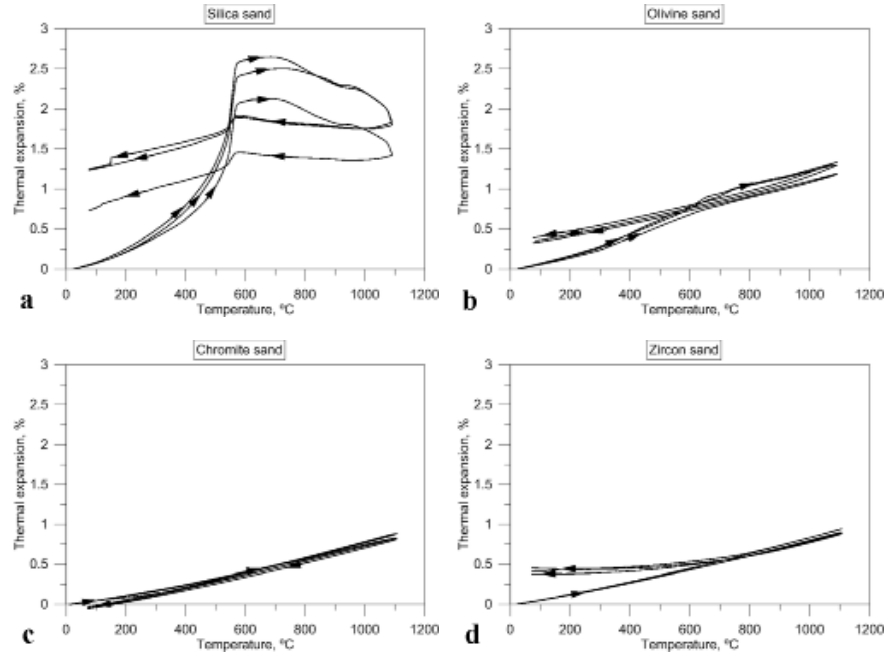

Fig. 3. Thermal expansion of silica (a), olivine (b), chromite (c) and zircon (d) sands versus temperature

therefore the strength of the sample decreases. When the binder structure becomes too weak the specimen collapses under its own weight. Degradation time is determined consistently at $-0,2 \mathrm{~mm}$ deformation.

According to the results (Fig. 4a-d), samples made of phenolic resins showed typically higher deformation and longer degradation times for of all types of refractories. On the other hand, the faster decomposition process of furan sand [17] ensures good shake-out properties after solidification, but the strength of the core is stable only for a given period. Horizontal parts in the curves indicate equilibrium between the thermal expansion of the basic refractory and the decomposition process of the binder system. However, in case of zircon sand (Fig. 4d), there is an interval between 10 and 20 seconds, when the deformation of the furan samples is slightly higher compared to specimens made with the phenolic resin. This effect is contradictory to the general behaviour of the phenolic resin in the other three mixtures, being more flexible compared to the furan binder during the overall heating process. Moreover, despite having almost
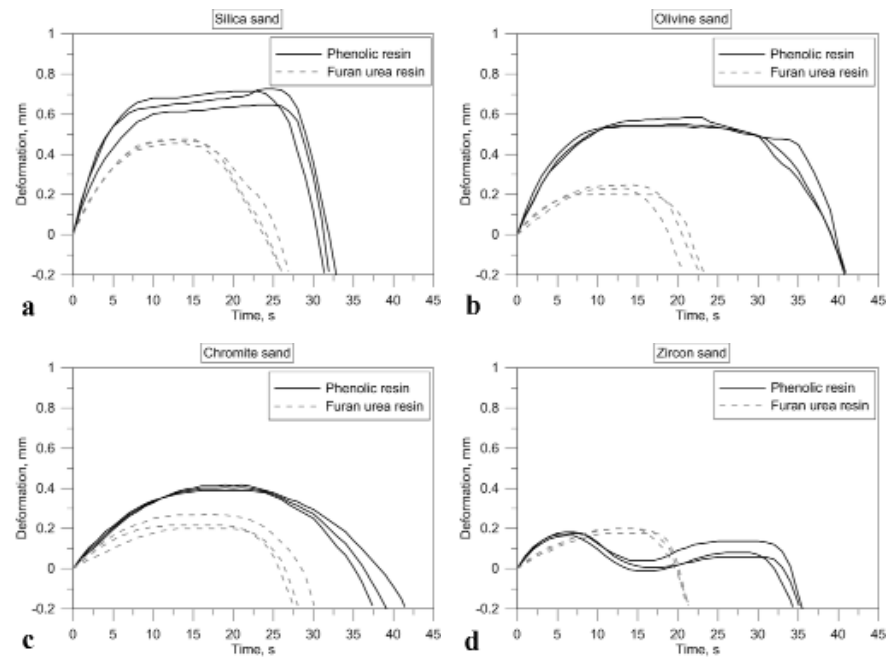

Fig. 4. Hot deformation of silica (a), olivine (b), chromite (c) and zircon (d) sand samples bonded with phenolic and furan urea binder 
identical thermal expansion profiles (Fig. 3b,d)), samples containing olivine and zircon sand show significantly different hot distortion characteristics. Various hot deformation characteristics of different phenolic bonded samples are also visible. These indicate, that the thermo-mechanical effects in the specimen are strongly affected by the type of sand.

The relationship between the results of unbonded and resin bonded samples are summarized in (Figs. 5,6), showing the maximum thermal expansion and the maximum deformation values of the studied refractories. The difference in the thermal expansion values of various sand types correlates well to the hot distortion results of bonded mixtures regardless of the type of resin. For example, the unbonded silica has the highest maximum dilatation, accordingly furan urea and phenolic resin bonded silica samples have the highest deformation, and zircon has the lowest. Although, in case of olivine and chromite sand, the results of the furan resin mixtures proves much better correlation.

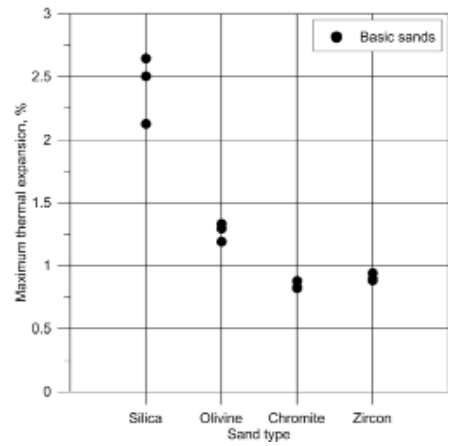

Fig. 5. Maximum thermal expansion of the studied (different) refractories

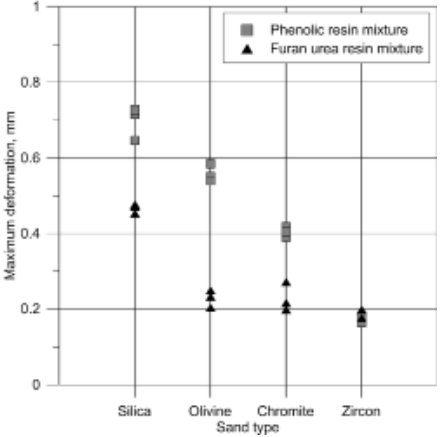

Fig. 6. Maximum hot deformation of phenolic and furan urea resin bonded samples

\section{Conclusions}

In this study, the thermal expansion of four types of foundry sands was measured during heating up and also during cooling down to reproduce temperature relations of the casting process. The hot distortion of resin bonded mixtures made by the investigated aggregates was also studied in order to evaluate the relation between the thermal dilation of the sands and the hot deformation of the moulding mixtures.

The results showed, that the thermal expansion of foundry sand largely influences the hot distortion behaviour, but depend- ing on the type of binder used. The maximum distortion of furan urea resin bonded samples corresponds better to the maximum expansion values of the unbonded sand samples, compared to the phenolic resin bonded specimens. Phenolic resin bonded specimens expressed significantly different hot deformation characteristics in case of olivine and zircon, despite these sands have almost identical thermal expansion curves.

Interpretation of distortion results is complicated because the decomposition process of the binder bridges and the expansion of the basic sand occurs simultaneously and the core can lose its strength faster, than the effect of the expansion occurs and collapses before the refractory reaches its maximum expansion. Thermophysical and physical properties of the basic sands other than thermal dilatation as the subjects of future investigations can support the understanding of the processes taking place at elevated temperatures.

\section{Acknowledgements}

The present work was financed by the Swedish Knowledge Foundation. Cooperating parties in the project are Jönköping University, Scania CV AB and Volvo Powertrain Production Gjuteriet AB. Participating persons from these institutions/companies are acknowledged.

\section{REFERENCES}

[1] R.A. Kleiv, Int. J. Min. Met. Mater. 19 (3), 185 (2012).

[2] M. Břuska, J. Beňo, M Cagala, V Jasinková, Arch. Foundry Eng. 12 (2), 9-14 (2012).

[3] J R. Brown, Foseco Ferrous Foundryman's Handbook (2000).

[4] S. Hass, Giesserei lexikon, p. 704. (2008).

[5] American Foundry Society, Mold \& Core Test Handbook, $4^{\text {th }}$ edition, 2014.

[6] J. Thiel, M. Ziegler, P. Dziekonski, S. Joyce, Trans. Amer. F. 117, 383-400 (2007).

[7] S.I. Bakhtiyarov, R.A. Overfelt, D. Wang, Int. J. Thermophys. 26 (1), 141-149 (2005).

[8] F. Peters, R. Voigt, S.Z. Ou, C. Beckermann, Int. J. Cast Metal. Res. 20 (5), 275-287 (2007).

[9] Z. Ignaszak, P. Popielarski, Defect Diffus. Forum 283-286, 382387 (2009).

[10] Z. Ignaszak, J.B. Prunier, Arch. Foundry Eng. 13, 30-36 (2013).

[11] Z. Ignaszak, P. Popielarski, T. Strek, Defect Diffus. Forum 312-315, 764-769 (2011).

[12] Z. Ignaszak, Arch. Foundry Eng. 76 (4), 69-76 (2010).

[13] S. McIntyre, S.M. Strobl, Foundry Manage. Technol. 126 (3), 22-27 (1998).

[14] J. Jakubski, S.M. Dobosz, Arch. Metall. Mater. 52 (3), 421-427 (2007).

[15] S.M. Dobosz, D. Drozynski, J. Jakubski, K. Major-Gabrys, Arch. Metall. Mater. 59 (3), 1093-1096 (2014).

[16] S.M. Dobosz, A. Grabarczyk, K. Major-Gabryś, J. Jakubski, Arch. Foundry Eng. 15 (2), 9-12 (2015).

[17] J. Zych, J. Mocek, Arch. Foundry Eng. 15 (4), 95-100 (2015). 